\title{
Development of a Virtual Reality Simulation for Remote Controlled Container Crane Operator Training
}

\author{
Aviral Goel \\ Ali Humaid Abdullah Al Tubi \\ Alaa Ahmed Eltayeb
}

\author{
Fontys University of Applied Science, Eindhoven \\ Fontys University of Applied Science, Eindhoven \\ Fontys University of Applied Science, Eindhoven
}

\begin{abstract}
Any large scale cargo port requires the use of container cranes for loading of intermodal containers as well as unloading them. The operator of such a large crane must be appropriately trained to prevent any accidents at the cargo port. This should be achieved by training crane operators on all the protocols of crane operation with significant hours of practice. The aim of this project is to develop a virtual reality (VR) simulation for operator training of container cranes which are remotely controlled, situated at the cargo ports. The simulation consists of a virtual control room equipped with joysticks and display screens, container crane, container ships, trucks and a cargo port environment. We used an actual super post-Panamax quay crane as a reference model for this simulation. The project has been built using Unity3d for the programming and 3DSMAX for the designing of models. The virtual joysticks in the simulation can be interacted by using the Oculus Rift VR setup thus allowing to maneuver the crane and the container load in all three dimensions. This paper mainly discusses the structure and implementation of the virtual reality simulation for crane operator training, along with a proposal for further improvements.
\end{abstract}

\section{Introduction}

The emergence of Virtual Reality (VR) technologies provides interactive simulation-based tools for training personnel for their work-related activities or health and safety. VR is increasingly catching the eye of agencies, large companies, and organization for recreating situations which put employees or the company at high risk of damage to property and life. The several advantages of VR simulator in providing training, has motivated large companies to increase the use of such systems in the constructions, medicine, marine sector (Ji-Liang et al., 1997; Mourant et al., 1998; Huang, 2003; A.H.Nayfeh, 2004; P. M, 2005; Korkealaakso et al., 2007).

Recently. The rise of Virtual Reality technology as a lucrative alternative for proving training stems from its ease of development and ease of coupling it with a variety of input devices, for instance, joysticks and motion sensors. Virtual Reality based simulations can also be developed by small teams and offer intricate interactable experiences that are progressively becoming close to real life.

VR has the capability of stimulating the brain to provide a close to the real-life virtual environment which can make the user feel reality in the digital world. By the use of the virtual reality equipment kit or it's input devices the system is able to replicate user interaction in real-world to the virtual world in realtime. Commonly used VR devices are the virtual reality headset, the controller and the motion sensors. Consequently, VR is able to deliver practical training which sometimes may not be possible to provide in real life due to complex situations such as bad weather, unavailability of machinery or operating cost. (Frank, P et al. 1999).

The training of high-quality personnel's for operating large container crane found at container terminals for loading and unloading intermodal containers from container ships is time-intensive, 


\section{Journal of Student Research}

Fourth Middle East College Student Research Conference, Muscat, Sultanate of Oman

expensive, and potentially hazardous. The crane system at the pivot of this development project is remote-controlled ship-to-shore (STS), often called Post-Panamax and Super-post-Panamax. PostPanamax and Super-post-Panamax types of container cranes can weigh between the range of 1,600-2,000 tons. Their sheer size results in requiring a significant amount of practice to attain a skill set that is appropriate for safe and efficient operating of a crane as an operator.

The process of training skilled personnel as a crane operator is a costly activity in terms of time and money, the process carries several costs and five major challenges to be faced. (CIBB, 2018).

The operator must learn to load and unload containers of varying mass with in a tolerable manner without causing damage to property and to correctly position them within a limited amount of time. The expenses include the opportunity cost of using the crane for training purposes and the resources required to oversee the functioning of the crane of this magnitude. The hazard results from an unskilled operator incorrectly maneuvering the crane and damaging the docked container ship, the containers or other nearby heavy machinery. The accident caused can be catastrophic and fatal to human lives. (CIBB, 2018). Although advantages of using VR are numerous in the training process, the availability of a comprehensive system focusing on the demand of the logistics and maritime crane-related sectors for training activities on very large remote-controlled STS cranes is severely limited.

Furthermore, the designing process for a virtual environment is a complex task. Choosing an adequate software and hardware framework is also not trivial. To address these problems, we have developed a Virtual Reality Crane Training Simulation.

Game engines have increasingly become the leading platform for developing and deploying most virtual reality projects.0 (Farouk, et al., 2015). This project was developed using the Unity Development Platform (Unity3D). It is the \#1 game development tool with the support to develop and build the games for Android, iOS, Windows, macOS, and the web. (Farouk, et al., 2015). The android build support along with Oculus integration streamlines the process for deploying VR hardware compatible applications. As per the analysis of available game engines, Unity3D, now called Unity Development Platform hails as the most popular game development engine, it is b was supported by its popularity, it dominates the market in terms of usage by game developers at $47 \%$ Game development in Unity3D uses the component model where one core class is aware of all possible components in the project which is a bit different approach than Object Oriented Programming (OOP), this provides a programming architecture which can scaled seamlessly (Farouk, et al., 2015).

This simulation was created using the Unity Development Platform (Unity3D) and integrated with the Oculus Rift VR headset, Oculus Touch controllers. Oculus is an immersive virtual reality system with two controllers and two sensors for tracking movement in real-time.

\section{Related Works}

Examples of immersive environments for training have been deployed in several fields, being applied in activities as hydraulic carry deck cranes (A. K . George et al, 2016), bridge crane training (Haoming Dong et al, 2009), surgical operation (Seymour NE, et al, 2002).

Angelov et al. present a non-immersive 3D and desktop-based system for training in power systems teaching. The author records the benefits of using virtual reality 3D models for an interactive presentation and its effects on the education process and retention of knowledge. (Angelov, 2007). Rojo et al., in their paper present the development of a virtual reality trainer for the sole purpose of assisting patients of Asperger's syndrome with interacting and presenting in a public environment without supervision (Rojo et al, 2019). 


\section{Journal of Student Research}

Fourth Middle East College Student Research Conference, Muscat, Sultanate of Oman

Some other applications of VR technology can be found in several other fields. (Buriol et al., 2019; Pulijala et al., 2018; Pellet et al., 2019).

\section{Training System Description}

The simulation is designed to familiarize new crane operators with the control room environment, it's control board system, maneuvering of the crane and optimal use of the tools available to the operator in the control room. Within this premise, the trainee should be able to learn how to obtain the maximum performance results from the crane.

The training system comprises four main elements:

1. Oculus VR Setup

2. Control Room Environment

3. Virtual Joystick Control System

4. Container Crane

The project was developed using the Unity3D game engine and C\# as the primary scripting language. 3DSMax is the 3D modeling software along with other tools such as Quixel and headus UVLayout. The simulation was modeled after the Super-post-Panamax types of container cranes which are remotely controlled. The crane and container models are based on the blueprints provided by the manufacturer. The design and specifications of the control room and control board are also based on the data and pictures provided by the port of Sohar, Sultanate of Oman.

\section{Oculus Virtual Reality Setup}

This simulation was developed using Unity3D game engine, and the Oculus Integration plugin for using the Oculus Rift. The simulation application runs on the desktop connected with Oculus Rift. During the simulation, the input from the user is collected by the virtual reality input devices, specifically one head-mounted display (HMD) two Oculus Touch controllers and two motion sensors that reproduce the translation and rotation of the player from the real world into the simulation. (Figure 1) 


\section{Journal of Student Research}

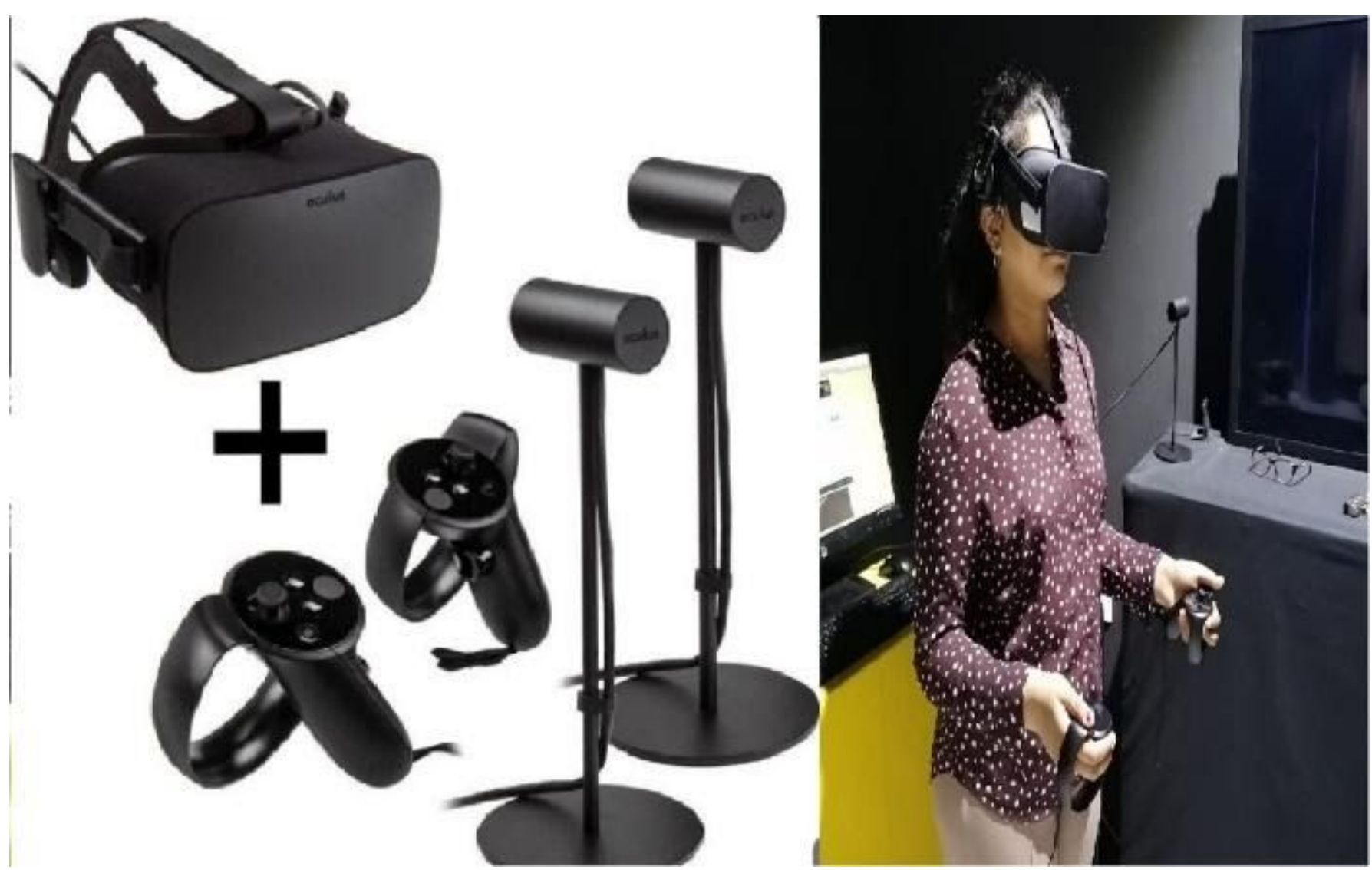

Figure 1. Oculus Components (left) | Person wearing HMD and controllers (right)

The controllers let the simulation replicate the illusion of grabbing and releasing the virtual joysticks in the real world. The buttons on the controller work for assisting the trainee in loading and unloading of the containers.

\section{Control Room Environment}

The Panamax and Super-post-Panamax types of crane considered for this simulation project are being controlled remotely from control rooms situated far away from the cranes at the ports.

(Figure 2). 


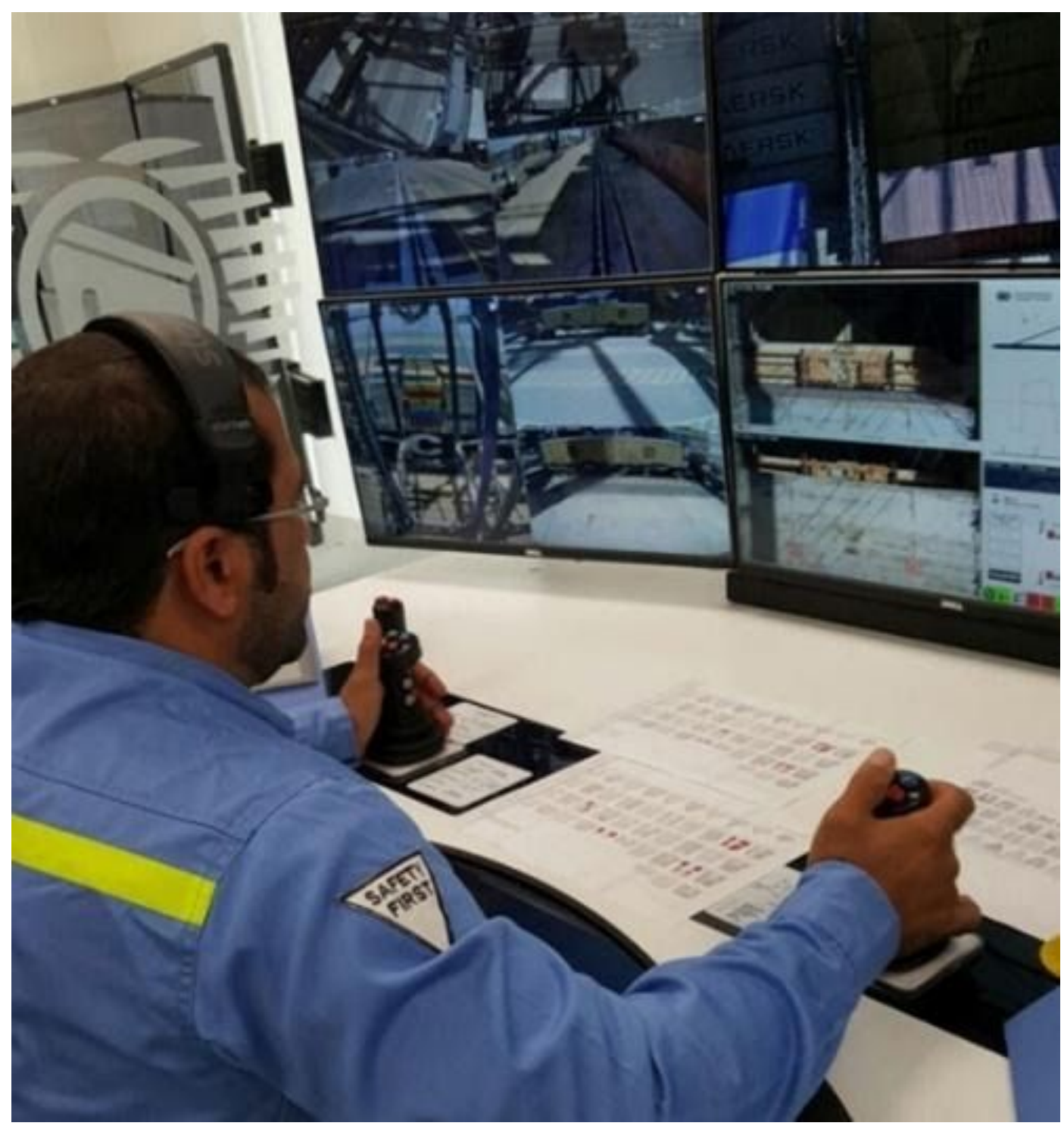

Figure 2. Operator control desk

The control room comprises of various monitor screens which stream live from the cameras installed on the crane, joysticks to control the crane movement, and various function buttons that can be used for the lock-and-load mechanism of the crane. spreader. The operators must maneuver and supervise the crane by using the control board and observing the crane from different angles provided on the screen. (Figure 3)

Figure 3. Virtual Control Room

The specifications and design of the control room have been taken from a real control room Sohar Port, Oman.

\section{Virtual Joystick Control System}

The virtual control board comprises of two joysticks, one touch screen and several buttons all inside the 3D environment. The joysticks and the touch screen are situated on the desk in front of the monitor screens. They can be interacted in much the same way as in real life using the hand position and hand movement of the trainee. This is a crucial part of the simulation as we have 
replicated the real functionality of the control board to the highest degree. The movement of the two joysticks allows for the maneuvering of the crane. (Figure 4)

Figure 4. point-of-view of the trainee for controlling the crane

As part of the training, the operator must learn to swiftly maneuver the crane using the joysticks inside the virtual reality simulation. He must also make sure that the crane spreader is positioned correctly for loading and unloading of the container by observing the crane from different perspectives provided on the monitor screens.

\section{Container Crane}

The crane model is scaled appropriately using the dimensions provided by the manufacturer. (Figure 5) It is maneuvered by the joysticks and the buttons provided in the control room. The spreader of the crane can be moved horizontally and vertically using the joysticks furnished in the control room whereas the entire frame of the crane can also be moved sideways as per the constraints. The locking mechanism of the spreader to grab and release containers is activated with buttons on the virtual joysticks. It is to be noted that Unity's physics engine with some additional modifications simulates the crane movement according to the mass of the container making very heavy or very light containers difficult to maneuver just as they would be in real-life scenarios.

Figure 5. Crane blueprint (left) | Crane model (right)

\section{Conclusion}

This virtual reality simulation is aimed to create a superior training experience than the conventional approach. The primary goal is to offer a solution which not only reduces the training costs but also completely eliminates the risk of any small or big accident. Due to the huge size of the crane and their large cost of operation, there is a lack in the number of training centers for operators. Our developed simulation uses the latest virtual reality technologies available. It also closely replicates the crane controls, crane movement, and port environment. This provides a better training experience and gives the opportunity to practice so that the operator can practice again and again until he understands the concepts.

\section{Future Scope}

The simulation can further be improved in terms of refining its current functionality as well as extending. To offer as much as effective training, we plan to implement a series of modules, namely

1. Performance Evaluation Report: To analyze the acumen of the trainee, a performance report can be generated for the instructor with an objective to better asses the trainee's skills.

2. Weather Manager: A wide range of simulated weather conditions and their effects on the operation of the crane, will help in further lifelike experience.

3. Extend the simulation experience by incorporating variety of very large size cranes.

4. Disaster response plan: A trainee can able be trained through the basic protocols of operating a crane in case of any mishap, such as emergency shutdown.

\section{Acknowledgments}

We are thankful to the Sas Centre for Virtual Reality, Ministry of Technology and Communication, 
Sultanate of Oman for their continuous support and technical facilities provided to us.

\section{References}

A. K. George, ML McLain, K Bijlani, R Jayakrishnan, R. R Bhavani, A Novel Approach for Training Crane Operators, 2016 IEEE 8th International Conference on Technology for Education

Angelov, A. N.; Styczynski, Z. A. Computer-aided 3D Virtual Training in Power System Education. In: IEEE Power Engineering Society General Meeting, 2007, p.1-4.

Buriol, Tiago \& Rosendo, Matheus \& de Geus, Klaus \& Scheer, Sergio \& Felsky, Carlos. (2019). A virtual reality training platform for live line maintenance of power distribution networks.

CIBB, 2018. Lack of Crane Safety Training: The Hidden Costs and the 5 Major Challenges in the Crane Industry. Retrieved from https://cicb.com/lack-of-crane-safety-training-the-hidden-costs-andthe-5-major-challenges-in-the-crane-industry/

A.H. Nayfeh and Daqaq, M. (2004). A virtual environment for ship-mounted cranes. International Journal of Modelling and Simulation, 24(4):272-279.

Farouk Messaoudi, Gwendal Simon and Adlen Ksentini, "Dissecting Game Engines: the Case if Unity3D", International Workshop on Network and Systems Support for Games (NetGames), 2015

Frank, P. Using simulations to provide safety and operations training in the electric power industry. In: Power Engineering Society 1999 Winter Meeting, IEEE. P.986-988 vol.2

Haoming Dong, Shaojun, Zou, Dingfang Chen, and Hongliu Xu, Research and Development of Distributed Bridge Crane Training System Based on Virtual Reality, 2nd International Conference on Intelligent computing technology and automation, 10-11 October 2009

Huang, J.-Y. (2003). Modeling and designing a low-cost high-fidelity mobile crane simulator. International Journal of Human-Computer Studies, 58(2):151- 176.

Ji-Liang, Jiing-Yih, L., D., H., J.-R., Ming-Chang, J., and G., C.-Y. (1997). Development of a virtual simulation system for crane-operating training. In Proceedings of ASME.

Jingming XIE," Research on Key Technologies Base Unity3D Game Engine", 7th International Conference on Computer Science and Education, 14-17 July 2012

P.M., Korkealaakso, Rouvinen, A.J., Moisio, S.M., and Peusaari, J. K. (2007). Development of a realtime simulation environment. Multibody System Dynamics, 17:177-194

Mourant, Wilson, B., R., Li, M., and Xu, W. (1998). A virtual environment for training overhead crane operators: real-time implementation. IIE Transactions, 30:589-595

Pulijala, Yeshwanth \& Ma, Minhua \& Pears, Matt \& Peebles, David \& Ayoub, A. (2018). An innovative virtual reality training tool for orthographic surgery. International Journal of oral and maxillofacial surgery. 47. 10.1016/j.ijom.2018.01.005.

Pulijala, Yeshwanth \& Ma, Minhua \& Pears, Matt \& Peebles, David \& Ayoub, A. (2018). An innovative virtual reality training tool for orthognathic surgery. International Journal of oral and maxillofacial surgery. 47. 10.1016/j.ijom.2018.01.005.

Rojo, Diego \& Mayor, Jesus \& García Rueda, Jose Jesus \& Raya, Laura \& Potel, Mike. (2019). A 


\section{Journal of Student Research}

Fourth Middle East College Student Research Conference, Muscat, Sultanate of Oman

Virtual Reality Training Application for Adults with Asperger's Syndrome. IEEE computer graphics and applications. 39. 104-111. 10.1109/MCG.2018.2884272.

Rouvinen, A. (2005). Container gantry crane simulator for operator training. In Publishing, P. E., editor, Proceedings of the Institution of Mechanical Engineers, Part K: Journal of Multi-body Dynamics, volume 219, pages 325-336

Seymour NE, Gallagher AG, Roman SA, et al. Virtual reality training improves operating room performance: results of a randomized, double-blinded study. Ann Surg. 2002;236(4):458-464. doi:10.1097/00000658-200210000-00008 